\title{
Extended Camassa-Holm Hierarchy and Conserved Quantities
}

\author{
Rossen I. Ivanov ${ }^{1}$ \\ School of Mathematics, Trinity College, \\ Dublin 2, Ireland \\ Tel: $+353-1-6082898$; \\ Fax: + 353 - 1- 608 2282; E-mail: ivanovr@tcd.ie
}

\begin{abstract}
An extension of the Camassa-Holm hierarchy is constructed in this letter. The conserved quantities of the hierarchy are studied and a recurrent formula for the integrals of motion is derived.
\end{abstract}

PACS: 02.30.Ik; 05.45.Yv; 45.20.Jj; 02.30.Jr.

Key words: Conservation Laws; Lax Pair; Integrable Systems; Solitons.

\footnotetext{
${ }^{1}$ On leave from the Institute for Nuclear Research and Nuclear Energy, Bulgarian Academy of Sciences, Sofia, Bulgaria.
} 


\section{Introduction}

The Camassa-Holm equation $(\mathrm{CH})$ [1]

$$
u_{t}-u_{x x t}+2 \omega u_{x}+3 u u_{x}-2 u_{x} u_{x x}-u u_{x x x}=0,
$$

where $\omega$ is a real constant parameter, describes the unidirectional propagation of shallow water waves over a flat bottom 1, 2. It firstly appeared in [3] as an equation with a bi-hamiltonian structure. $\mathrm{CH}$ is a completely integrable equation [4, 5, 6, 7, 8, 9, describing permanent and breaking waves 10, 11. Its solitary waves are stable solitons if $\omega>0$ [12, 13, 14, 15] or peakons if $\omega=0$ [1, 16]. CH arises also as an equation of the geodesic flow for the $H^{1}$ metrics on the Bott-Virasoro group [17, 18, 19]. The bi-Hamiltonian form of (11) is [1, 3, 5.

$$
m_{t}=-\left(\partial-\partial^{3}\right) \frac{\delta H_{2}[m]}{\delta m}=-(2 \omega \partial+m \partial+\partial m) \frac{\delta H_{1}[m]}{\delta m}
$$

where

$$
m=u-u_{x x},
$$

and the Hamiltonians are

$$
\begin{aligned}
& H_{1}[m]=\frac{1}{2} \int m u \mathrm{~d} x, \\
& H_{2}[m]=\frac{1}{2} \int\left(u^{3}+u u_{x}^{2}+2 \omega u^{2}\right) \mathrm{d} x .
\end{aligned}
$$

The integration is from $-\infty$ to $\infty$ in the case of Schwartz class functions, and over one period in the periodic case.

In [20] it is shown that $\mathrm{CH}$ has an infinite number of local conserved quantities. A scheme for computation of the conservation laws is proposed in [21, 22, 23]. In this contribution we present a scheme, providing an explicit recurrent formula for the infinite sequence of independent integrals of motion for a chain of $\mathrm{CH}$ type equations.

The equation (11) admits a Lax pair [1]

$$
\begin{aligned}
\Psi_{x x} & =\left(\frac{1}{4}+\zeta(m+\omega)\right) \Psi \\
\Psi_{t} & =\left(\frac{1}{2 \zeta}-u\right) \Psi_{x}+\frac{u_{x}}{2} \Psi .
\end{aligned}
$$

Recently, various multi-component generalizations of the $\mathrm{CH}$ equation are under intense investigation, e.g. 9, 24, 25, 26, 27] and probably some others.

In this work we also present a construction for a multi-component system, which admits reduction to $\mathrm{CH}$ equation. 
To this end, instead of the Lax pair (6), (77), we consider a more general one, leading to a hierarchy of Camassa-Holm type:

$$
\begin{aligned}
\Psi_{x x} & =Q(x, \lambda) \Psi \\
\Psi_{t} & =-U(x, \lambda) \Psi_{x}+\frac{1}{2} U_{x}(x, \lambda) \Psi
\end{aligned}
$$

where

$$
\begin{aligned}
Q(x, \lambda) & =\lambda^{n} q_{n}(x)+\lambda^{n-1} q_{n-1}(x)+\ldots+\lambda q_{1}(x)+\frac{1}{4}, \\
U(x, \lambda) & =u_{0}(x)+\frac{u_{1}(x)}{\lambda}+\ldots \frac{u_{k}(x)}{\lambda^{k}} .
\end{aligned}
$$

The compatibility condition of (8), (9) gives the equation

$$
Q_{t}=\frac{1}{2} U_{x x x}-2 U_{x} Q-U Q_{x},
$$

which, due to (10), (11), is equivalent to a chain of $n$ evolution equations with $k+1$ differential constraints for the $n+k+1$ variables $q_{1}, q_{2}, \ldots, q_{n}, u_{0}$, $u_{1}, \ldots, u_{k}$ ( $n$ and $k$ are arbitrary natural numbers, i.e. positive integers):

$$
\begin{array}{rlr}
q_{n-r, t} & =-\sum_{s=\max (0, r-k)}^{r}\left(2 u_{r-s, x} q_{n-s}+u_{r-s} q_{n-s, x}\right), \quad r=0,1, \ldots, n-1, \\
0 & =\frac{1}{2}\left(u_{r, x x x}-u_{r, x}\right)-\sum_{s=1}^{\min (n, k-r)}\left(2 u_{r+s, x} q_{s}+u_{r+s} q_{s, x}\right), \\
0 & =\frac{1}{2}\left(u_{k, x x x}-u_{k, x}\right) . & r=0,1, \ldots, k-1,
\end{array}
$$

The system (13) is similar to the hydrodynamic chain, studied in a series of papers [28, 29, 30, 31, and to other $\mathrm{CH}$ generalizations $9,24,26,27,32$. Let us now consider the following examples.

Example 1: $k=n=2$.

The choice $u_{2}=-1 / 2$ solves automatically one of the constraints. The other two differential constraints can be easily integrated, giving

$$
\begin{aligned}
& q_{1}=u_{1}-u_{1, x x}+\omega_{1}, \\
& q_{2}=u_{0}-u_{0, x x}+3 u_{1}^{2}-u_{1, x}^{2}-2 u_{1} u_{1, x x}+4 \omega_{1} u_{1}+\omega_{2},
\end{aligned}
$$

where $\omega_{1,2}$ are arbitrary constants. The system of equations for $u_{0}, u_{1}$ is 


$$
\begin{aligned}
& q_{2, t}+2 u_{0, x} q_{2}+u_{0} q_{2, x}=0, \\
& q_{1, t}+2 u_{0, x} q_{1}+u_{0} q_{1, x}+2 u_{1, x} q_{2}+u_{1} q_{2, x}=0 .
\end{aligned}
$$

Example 2: $k=1, n=2$.

This system was studied in more details in 25]. In the notations $u_{0} \equiv u$, $q_{1} \equiv-q$ and $q_{2} \equiv \rho^{2}$, and with the choice $u_{1}=-1 / 2$, the system can be written in the form

$$
\begin{aligned}
& q_{t}+u q_{x}+2 q u_{x}-\rho \rho_{x}=0, \\
& \rho_{t}+(u \rho)_{x}=0,
\end{aligned}
$$

where $q=u-u_{x x}+\omega$ and $\omega$ is an arbitrary constant.

Example 3: $\mathrm{CH}$ equation

$\mathrm{CH}$ can be considered as a reduction from the system (14) - (17). Indeed, one can obtain an integrable reduction of (14) - (17) by taking $u_{1}=\omega_{1}=0$. Then $q_{1}=0, q_{2}=u_{0}-u_{0, x x}+\omega_{2}$ and (16) is exactly the $\mathrm{CH}$ equation (1) with $u \equiv u_{0}$ and $\omega \equiv \omega_{2}$. Equation (17) is trivially satisfied. Thus, the $\mathrm{CH}$ corresponds to a Lax pair with

$$
Q(x, \lambda)=\lambda^{2} q_{2}(x)+\frac{1}{4}, \quad U(x, \lambda)=u_{0}(x)-\frac{1}{2 \lambda^{2}} .
$$

It is not difficult to recover the Lax pair (6) - (7) by identifying $\zeta=\lambda^{2}$.

$\mathrm{CH}$ can also be obtained as a reduction from (18) - (19) by setting $\rho=0$.

\section{Generating Function for the Integrals of Motion}

Introducing

$$
p=\frac{\Psi_{x}}{\Psi}
$$

from (8) we obtain (cf. 33, 34, 21])

$$
p_{x}+p^{2}=Q(x, \lambda) .
$$

Then, from (91), (21) and (22) the following conservation law follows:

$$
p_{t}=\left(\frac{1}{2} U_{x}-p U\right)_{x}
$$


Therefore $p(x, \lambda)$, regarded as a solution of (22), is the density of the generating function of the conservation laws. We notice that the densities are determined up to a constant. Indeed, if $\alpha(\lambda)$ is an arbitrary function of $\lambda$, the quantity $P=p+\alpha$ is also a generating function, since it satisfies

$$
P_{t}=\left(\frac{1}{2} U_{x}-P U+\alpha U\right)_{x} .
$$

We can use this freedom to fix the convergency properties of the integrals representing the conserved quantities.

Clearly, $p$ is related to the scattering matrix [7] when the Schwartz class of solutions is considered, or to the monodromy matrix in the periodic case [34.

Now it is evident that (23) or (24) represent a parameter-dependent conservation law, which is equivalent to a sequence of infinitely many conservation laws.

Indeed, since $\lambda$ is an arbitrary (spectral) parameter, one can expand the solution $p(x, \lambda)$ of (22) about $\lambda=\infty$. Let us suppose for simplicity that $n=2 a$ is an even number. (The case when $n$ is odd is handled in a similar manner, e.g. by introducing a new spectral parameter $\zeta$, such that $\lambda=\zeta^{2}$, see the $\mathrm{CH}$ case 21, 23], where $n=1$ ). Then

$$
p(x, \lambda)=p_{a} \lambda^{a}+\ldots+p_{1} \lambda+p_{0}++\sum_{s=1}^{\infty} \frac{p_{-s}}{\lambda^{s}} .
$$

There are finitely many positive powers in $\lambda$ due to their presence in the RHS of (22). From (23) and (25) the following infinite sequence of conservation laws follows (it is assumed $u_{r} \equiv 0$ for $r>k$ ):

$$
\begin{aligned}
& p_{a-r, t}=-\left(\sum_{s=0}^{r} u_{r-s} p_{a-s}\right)_{x}, \quad r=0,1, \ldots, a-1, \\
& p_{-r, t}=\left(\frac{1}{2} u_{r, x}-\sum_{s=0}^{a+r} u_{a+r-s} p_{a-s}\right)_{x}, \quad r=0,1, \ldots .
\end{aligned}
$$

Let us now expand the solution $p(x, \lambda)$ of (22) about $\lambda=0$ :

$$
p(x, \lambda)=p_{0}+\sum_{s=1}^{\infty} p_{s} \lambda^{s} .
$$

Note that the expansion coefficients $p_{s}$ in (27) are not the same as those in (25). From (23) and (27) another infinite sequence of conservation laws follows: 


$$
\begin{aligned}
& p_{r, t}=-\left(\sum_{s=0}^{k} u_{s} p_{s+r}\right)_{x} \quad r=1,2, \ldots, \\
& p_{0, t}=\left(\frac{1}{2} u_{0, x}-\sum_{s=0}^{k} u_{s} p_{s}\right)_{x}
\end{aligned}
$$

and in addition, some of the constraints can be rewritten in the form:

$$
\left(\frac{1}{2} u_{r, x}-\sum_{s=0}^{k-r} u_{r+s} p_{s}\right)_{x}=0, \quad r=1,2, \ldots, k .
$$

In general, there are two different solutions of (22). However, these solutions do not represent independent integral densities of conserved quantities, due to the following result, showing that each of the solutions can be expressed linearly through the other one, up to an exact derivative:

Lemma 1 If $p^{+}(x, \lambda)$ and $p^{-}(x, \lambda)$ are two different solutions of (22), then there exists a function $f(x, \lambda)$, such that

$$
p^{+}(x, \lambda)=-p^{-}(x, \lambda)+f_{x}(x, \lambda) .
$$

Proof. Writing (22) for $p^{+}(x, \lambda)$ and $p^{-}(x, \lambda)$ and subtracting the two equations we obtain immediately $f(x, \lambda)=-\ln \left[p^{+}(x, \lambda)-p^{-}(x, \lambda)\right]$.

\section{Computation of the Conserved Quantities}

In order to find the integral densities, one needs to compute explicitly the expansion coefficients in (25), (27). In this Section we will illustrate the method at the system from Example 1. (The conservation laws for the $\mathrm{CH}$, Example 3, follow immediately via the described reduction.) Clearly, one can apply an analogous procedure to any particular case of the system (13).

Before going into the actual computations, the following observation is in order. Exactly as in [7, 35, using only (16) one can prove that $q_{2}(x, t)$ does not change sign if $q_{2}(x, 0)$ does not. The idea of proof is as follows. Consider the diffeomorphism of the line $\varphi(x, t)$, such that

$$
\varphi_{t}=u_{0}(\varphi(x, t), t), \quad \varphi(x, 0)=x .
$$

The solution of (31) is unique and represents an increasing diffeomorphism $\left(\varphi_{x}>0\right.$ for all $\left.t\right)$ of $\mathrm{R} \ni x$ [35]. Then, using (16) and (31), one can check that

$$
q_{2}(\varphi(x, t), t) \varphi_{x}^{2}(x, t)=q_{2}(x, 0)
$$


and the claim easily follows. Then, for simplicity, in order to make sense of the expressions like $\sqrt{q_{2}(x, t)}, 1 / \sqrt{q_{2}(x, t)}$, etc; we assume that the initial data is such that $q_{2}(x, 0)$ does not change sign, i.e. $q_{2}(x, 0)>0$.

Also, in the case when $u_{0}, u_{1}, \ldots$ are Schwartz class functions, it may happen that the integral density at $x \rightarrow \pm \infty$ is not zero but a constant. Then, in order to make sense of the integral, the density obviously should be reduced by the same constant, cf. (24).

The equation (22) is

$$
p_{x}+p^{2}=\frac{1}{4}+\lambda q_{1}+\lambda^{2} q_{2},
$$

and admits a solution of the form (25):

$$
p=p_{1} \lambda+p_{0}+\sum_{s=1}^{\infty} \frac{p_{-s}}{\lambda^{s}}
$$

where $p_{1}= \pm \sqrt{q_{2}}$, i.e. there are two solutions of (33). Since these two solutions do not produce independent integral densities due to the Lemma [1. we proceed with, say, $p_{1}=\sqrt{q_{2}}$. The first nontrivial integral from here is

$$
h_{1} \equiv \int p_{1} \mathrm{~d} x=\int \sqrt{q_{2}} \mathrm{~d} x
$$

From (33) and (34) we have

$$
2 p_{1} p_{0}+p_{1, x}=q_{1}, \quad p_{0}=\frac{q_{1}}{2 \sqrt{q_{2}}}-\frac{q_{2, x}}{4 q_{2}} .
$$

Neglecting the exact derivative, we obtain the integral

$$
h_{0}=\frac{1}{2} \int \frac{q_{1}}{\sqrt{q_{2}}} \mathrm{~d} x \text {. }
$$

The next equation,

$$
p_{0}^{2}+2 p_{1} p_{-1}+p_{0, x}=\frac{1}{4}
$$

gives

$$
p_{-1}=\frac{1}{32}\left(\frac{4}{\sqrt{q_{2}}}+\frac{q_{2, x}^{2}}{q_{2}^{5 / 2}}-\frac{4 q_{1}^{2}}{q_{2}^{3 / 2}}\right)+\left(\frac{q_{2, x}}{8 q_{2}^{3 / 2}}-\frac{q_{1}}{4 q_{2}}\right)_{x}
$$


and thus we obtain the integral

$$
h_{-1}=\frac{1}{32} \int\left(\frac{4}{\sqrt{q_{2}}}+\frac{q_{2, x}^{2}}{q_{2}^{5 / 2}}-\frac{4 q_{1}^{2}}{q_{2}^{3 / 2}}\right) \mathrm{d} x .
$$

It is not difficult to derive the general recurrent formula from (33) and (34):

$$
\begin{aligned}
h_{j} & =\int p_{j} \mathrm{~d} x, \quad j=1,0,-1, \ldots, \\
p_{1} & =\sqrt{q_{2}}, \quad p_{0}=\frac{q_{1}}{2 \sqrt{q_{2}}}-\frac{q_{2, x}}{4 q_{2}}, \quad p_{-1}=\frac{1}{2 p_{1}}\left(\frac{1}{4}-p_{0}^{2}-p_{0, x}\right), \\
p_{-j} & =-\frac{1}{2 p_{1}}\left(\sum_{i=0}^{j-1} p_{-i} p_{-j+i+1}+p_{-j+1, x}\right), \quad j \geq 2 .
\end{aligned}
$$

Now from (42), (43) and (23) one can express the conservation laws in differential form (26) with $a=1$ :

$$
\begin{aligned}
p_{1, t} & =-\left(u_{0} p_{1}\right)_{x} \\
p_{0, t} & =\left(\frac{1}{2} u_{0, x}-u_{0} p_{0}-u_{1} p_{1}\right)_{x} \\
p_{-1, t} & =\left(\frac{1}{2} u_{1, x}+\frac{1}{2} p_{1}-u_{1} p_{0}-u_{0} p_{-1}\right)_{x}, \quad j \geq 2 . \\
p_{-j, t} & =\left(\frac{1}{2} p_{-j+2}-u_{0} p_{-j}-u_{1} p_{-j+1}\right)_{x}, \quad j
\end{aligned}
$$

The reduction of (41) - (44) to the $\mathrm{CH}$ case (Example 3) is straightforward via $q_{1}=u_{1}=0$. All even densities, $p_{0}, p_{-2}, \ldots$ are exact derivatives and do not produce independent integrals of motion. Each of the remaining odd densities $p_{1}, p_{-1}, p_{-3}, \ldots$ produces only one independent integral of motion. Thus the $\mathrm{CH}$ integrals are $h_{1}, h_{-1}, h_{-3}, \ldots$.

Let us now take the expansion (27). Since in this case $u_{2}=-1 / 2$, from (29) for $r=k$ it follows that $p_{0, x}=0$ and then from (22), $p_{0}= \pm 1 / 2$. Due to the Lemma 1 we consider here only the first possibility, i.e. $p_{0}=1 / 2$ :

$$
p=\frac{1}{2}+\sum_{s=1}^{\infty} p_{s} \lambda^{s}
$$

Note that in (45) $p_{1}$ is not the same as in (34). From (33) and (45) we obtain

$$
p_{1}+p_{1, x}=q_{1}
$$


which due to (14) has a solution

$$
p_{1}=u_{1}-u_{1, x}+\omega_{1},
$$

leading to the integral $\left(h_{1}\right.$ is not the same as in (35) $)$

$$
h_{1}=\int u_{1} \mathrm{~d} x .
$$

The next equation from (33) and (45) is

$$
p_{2}+p_{2, x}+p_{1}^{2}=q_{2} .
$$

Using (15), (47) one can verify that

$$
p_{2}=u_{0}-u_{0, x}+2 u_{1}^{2}-2 u_{1} u_{1, x}+2 \omega_{1} u_{1}-\omega_{1}^{2}+\omega_{2} .
$$

Then the next independent integral is

$$
h_{2}=\int\left(u_{0}+2 u_{1}^{2}\right) \mathrm{d} x+2 \omega_{1} h_{1} .
$$

The equation for $p_{3}$ is

$$
p_{3}+p_{3, x}+2 p_{1} p_{2}=0
$$

giving

$$
h_{3}=\int p_{3} \mathrm{~d} x=-2 \int p_{1} p_{2} \mathrm{~d} x
$$

where $p_{1}$ and $p_{2}$ are given by (47), (50). We notice that formally (see (52))

$$
p_{3}=-\left(1+\partial_{x}\right)^{-1} 2 p_{1} p_{2} .
$$

The equation for $p_{4}$ is

$$
p_{4}+p_{4, x}+2 p_{1} p_{3}+p_{2}^{2}=0
$$

and thus 


$$
h_{4}=\int p_{4} \mathrm{~d} x=-\int\left(2 p_{1} p_{3}+p_{2}^{2}\right) \mathrm{d} x
$$

In order to express $\int p_{1} p_{3} \mathrm{~d} x$ only via the known $p_{1}$ and $p_{2}$ we proceed as follows, using (52), (47) and neglecting total derivatives:

$$
\begin{aligned}
& \int p_{1} p_{3} \mathrm{~d} x=-2 \int p_{1}^{2} p_{2} \mathrm{~d} x-\int p_{1} p_{3, x} \mathrm{~d} x= \\
& -2 \int p_{1}^{2} p_{2} \mathrm{~d} x-\int\left(u_{1}-u_{1, x}+\omega_{1}\right) p_{3, x} \mathrm{~d} x= \\
& -2 \int p_{1}^{2} p_{2} \mathrm{~d} x-\int\left(u_{1} p_{3, x}+u_{1, x}\left(2 p_{1} p_{2}+p_{3}\right)\right) \mathrm{d} x= \\
& -2 \int p_{1}^{2} p_{2} \mathrm{~d} x-\int\left(\left(u_{1} p_{3}\right)_{x}+2 u_{1, x} p_{1} p_{2}\right) \mathrm{d} x= \\
& -2 \int p_{1} p_{2}\left(u_{1, x}+p_{1}\right) \mathrm{d} x=-2 \int p_{1} p_{2}\left(u_{1}+\omega_{1}\right) \mathrm{d} x= \\
& -2 \int p_{1} p_{2} u_{1} \mathrm{~d} x+\omega_{1} h_{3} .
\end{aligned}
$$

Finally,

$$
h_{4}=\int\left(4 u_{1} p_{1} p_{2}-p_{2}^{2}\right) \mathrm{d} x-2 \omega_{1} h_{3} .
$$

The densities for the higher integrals are, in general, nonlocal.

To summarize: the conserved quantities are

$$
h_{j}=\int p_{j} \mathrm{~d} x, \quad j=1,2, \ldots,
$$

where according to (33) and (45) the integral densities $p_{j}$ can be computed recurrently:

$$
p_{j}=-\left(1+\partial_{x}\right)^{-1} \sum_{i=1}^{j-1} p_{i} p_{j-i}, \quad j \geq 3,
$$

where $p_{1}$ and $p_{2}$ are given in (47), (50), e.g. see (54). Again from (60) and (23), the conservation laws can be expressed in differential form (28):

$$
p_{j, t}=\left(\frac{1}{2} p_{j+2}-u_{1} p_{j+1}-u_{0} p_{j}\right)_{x}, \quad j=1,2, \ldots
$$


The reduction to $\mathrm{CH}$ (Example 3) goes as follows. With $q_{1}=u_{1}=\omega_{1}=$ 0 we have (47) $p_{1}=0$ and from (50), bearing in mind that in this case $u_{0} \equiv u, \omega_{2} \equiv \omega$,

$$
p_{2}=u-u_{x}+\omega .
$$

Then, clearly all odd integral densities are zero. Furthermore, $h_{2}$ gives the $\mathrm{CH}$ integral $H_{0}=\int m \mathrm{~d} x$ and $h_{4}$ leads to $H_{1}$ [recall (4)]. Now we can verify that the reduction of $h_{6}$ leads to the second $\mathrm{CH}$ Hamiltonian $H_{2}$ [recall (5)].

The equation for $p_{6}$ with the reduction is

$$
p_{6}+p_{6, x}+2 p_{2} p_{4}=0
$$

and correspondingly

$$
h_{6}=-2 \int p_{2} p_{4} \mathrm{~d} x .
$$

The equation for $p_{4}$, (55) now is

$$
p_{4}+p_{4, x}+p_{2}^{2}=0,
$$

and therefore, in order to compute $\int p_{2} p_{4} \mathrm{~d} x$ we proceed as follows. Multiplying (65) by $p_{2}$ and then using (65) and (62) we obtain:

$$
\begin{aligned}
& \int p_{2} p_{4} \mathrm{~d} x=-\int p_{2}^{3} \mathrm{~d} x-\int p_{2} p_{4, x} \mathrm{~d} x= \\
& -\int p_{2}^{3} \mathrm{~d} x-\int\left(u-u_{x}+\omega\right) p_{4, x} \mathrm{~d} x= \\
& -\int p_{2}^{3} \mathrm{~d} x-\int\left(u p_{4, x}+u_{x}\left(p_{2}^{2}+p_{4}\right)\right) \mathrm{d} x= \\
& -\int p_{2}^{3} \mathrm{~d} x-\int\left(\left(u p_{4}\right)_{x}+u_{x} p_{2}^{2}\right) \mathrm{d} x= \\
& -\int p_{2}^{2}\left(u_{x}+p_{2}\right) \mathrm{d} x=-\int p_{2}^{2}(u+\omega) \mathrm{d} x= \\
& -\int\left(u^{3}+u u_{x}^{2}+2 \omega u^{2}\right) \mathrm{d} x-3 \omega^{2} H_{0}-2 \omega H_{1},
\end{aligned}
$$

leading to the independent integral

$$
H_{2}=\frac{1}{2} \int\left(u^{3}+u u_{x}^{2}+2 \omega u^{2}\right) \mathrm{d} x .
$$

In other words, the $\mathrm{CH}$ conserved quantities are reproduced by the even integrals $h_{2}, h_{4}, h_{6}, \ldots$. 


\section{Acknowledgements}

The author is grateful to Prof. A. Constantin for helpful discussions and to an anonymous referee for important suggestions. The author also acknowledges funding from the Science Foundation Ireland, Grant 04/BR6/M0042.

\section{References}

[1] R. Camassa and D. Holm, Phys. Rev. Lett. 711661 (1993).

[2] R.S. Johnson, J. Fluid. Mech. 457, 63 (2002).

[3] A. Fokas and B. Fuchssteiner, Physica D4, 47 (1981).

[4] R. Beals, D. Sattinger, and J. Szmigielski, Adv. Math. 140, 190 (1998).

[5] A. Fokas, P.J. Olver, and P. Rosenau, in: Algebraic Aspects of Integrable Systems: In Memory of Irene Dorfman (Eds. A.S. Fokas and I.M. Gel'fand), Progress in Nonlinear Differential Equations, Vol. 26, Birkhauser, Boston 1996, p. 93.

[6] A. Constantin and H.P. McKean, Commun. Pure Appl. Math. 52, 949 (1999).

[7] A. Constantin, Proc. R. Soc. Lond. A457, 953 (2001).

[8] J. Lenells, J. Nonlin. Math. Phys. 9, 389 (2002).

[9] F. Gesztesy and H. Holden, Soliton Equations and Their AlgebroGeometric Solutions, Vol. I: (1+1)-Dimensional Continuous Models, Cambridge Studies in Advanced Mathematics, Vol. 79, Cambridge University Press, Cambridge 2003.

[10] A. Constantin and J. Escher, Acta Mathematica 181, 229 (1998).

[11] A. Constantin, Ann. Inst. Fourier (Grenoble) 50, 321 (2000).

[12] R. Beals, D. Sattinger, and J. Szmigielski, Inv. Problems 15, L1 (1999).

[13] A. Constantin and W. Strauss, Commun. Pure Appl. Math. 53, 603 (2000).

[14] A. Constantin and W. Strauss, J. Nonlin. Sci. 12, 415 (2002).

[15] R.S. Johnson, Proc. Roy. Soc. Lond. A 459, 1687 (2003).

[16] Y. A. Li, P.J. Olver, and P. Rosenau, in: Nonlinear Theory of Generalised Functions: Vienna 1997, Chapman \& Hall/CRC Res. Notes Math. 401, Chapman \& Hall/CRC, Boca Raton, FL 1999, p. 129. 
[17] G. Misiolek, J. Geom. Phys. 24, 203 (1998).

[18] A. Constantin and B. Kolev, Comment. Math. Helv. 78, 787 (2003).

[19] A. Constantin, T. Kappeler, B. Kolev, and P. Topalov, On Geodesic Exponential Maps of the Virasoro Group, Preprint No 13-2004, Institute of Mathematics, University of Zurich 2004.

[20] M. Fisher and J. Shiff, Phys. Lett. A 259, 371 (1999).

[21] E. Reyes, Lett. Math. Phys. 59, 117 (2002).

[22] J. Lenells, J. Phys. A: Math. Gen. 38, 869 (2005).

[23] P. Casati, P. Lorenzoni, G. Ortenzi, and M. Pedroni, J. Math. Phys. 46, 042704 (2005).

[24] D. Holm and Z. Qiao, Commun. Math. Phys. 239, 309 (2003).

[25] M. Chen, S.-Q. Liu and Y. Zhang , A 2-Component Generalization of the Camassa-Holm Equation and Its Solutions, preprint nlin.SI/0501028 (2005).

[26] P. Estévez and J. Prada, J. Phys. A: Math. Gen. 38, 1287 (2005).

[27] R. Kraenkel, M. Senthilvelan, and A. Zenchuk, J. Math. Phys. 41, 3160 (2000).

[28] L. Martínez Alonso and A. Shabat, Phys. Lett. A 300, 58 (2002).

[29] L. Martínez Alonso and A. Shabat, J. Nonlin. Math. Phys. 10, 229 (2003).

[30] L. Martínez Alonso and A. Shabat, Theor. Math. Phys. 140, 1073 (2004).

[31] A. Shabat, J. Nonlin. Math. Phys. 12, 614 (2005).

[32] P. Clarkson, P. Gordoa, and A. Pickering, Inv. Problems 13, 1463 (1997).

[33] A. Degasperis, D. Holm, and A. Hone, Theor. Math. Phys. 133, 1463 (2002).

[34] J.-L. Gervais, Phys. Lett. B 160, 277 (1985).

[35] A. Constantin, J. Math. Phys. 46, 023506 (2005). 